\section{OPEN ACCESS}

Edited by:

Cedric Bardy,

Flinders University, Australia

Reviewed by:

Bilal Ersen Kerman,

Istanbul Medipol University, Turkey

Li-Jin Chew,

Brown University, United States

*Correspondence:

Valéria de Almeida

val_farmac@yahoo.com.br

Daniel Martins-de-Souza

dmsouza@unicamp.br

Specialty section:

This article was submitted to Molecular Signalling and Pathways,

a section of the journal

Frontiers in Molecular Neuroscience

Received: 01 March 2021 Accepted: 27 April 2021

Published: 28 May 2021

Citation:

Falvella ACB, Smith BJ,

Silva-Costa $L C$, Valença $A G F$,

Crunfli F, Zuardi AW, Hallak JE, Crippa JA, Almeida V and

Martins-de-Souza D

(2021) Cannabidiol Displays

Proteomic Similarities to

Antipsychotics in Cuprizone-Exposed

Human Oligodendrocytic

Cell Line MO3.13.

Front. Mol. Neurosci. 14:673144.

doi: 10.3389/fnmol.2021.673144

\title{
Cannabidiol Displays Proteomic Similarities to Antipsychotics in Cuprizone-Exposed Human Oligodendrocytic Cell Line M03.13
}

\begin{abstract}
Ana Caroline Brambilla Falvella ${ }^{1}$, Bradley Joseph Smith ${ }^{1}$, Licia C. Silva-Costa ${ }^{1}$, Aline G. F. Valença ${ }^{1}$, Fernanda Crunfli ${ }^{1}$, Antonio W. Zuardi ${ }^{2}$, Jaime E. Hallak ${ }^{2}$, José A. Crippa ${ }^{2}$, Valéria de Almeida ${ }^{1 *}$ and Daniel Martins-de-Souza ${ }^{1,3,4,5 *}$
\end{abstract}

${ }^{1}$ Laboratory of Neuroproteomics, Department of Biochemistry and Tissue Biology, Institute of Biology, University of Campinas (UNICAMP), Campinas, Brazil, ${ }^{2}$ Department of Neurosciences and Behavior, Faculty of Medicine of Ribeirão Preto, University of São Paulo, Ribeirão Preto, Brazil, ${ }^{3}$ Instituto Nacional de Biomarcadores em Neuropsiquiatria (INBION) Conselho Nacional de Desenvolvimento Científico e Tecnológico, São Paulo, Brazil, ${ }^{4}$ Experimental Medicine Research Cluster (EMRC), University of Campinas, Campinas, Brazil, ${ }^{5}$ D'Or Institute for Research and Education (IDOR), São Paulo, Brazil

Cannabidiol, a compound of Cannabis sativa, has been proposed as an alternative treatment of schizophrenia. Preclinical and clinical data have suggested that cannabidiol shares more similarity with atypical antipsychotics than typical, both of which are customarily used to manage schizophrenia symptoms. While oligodendrocytes are known to be relevant targets of antipsychotics, the biochemical knowledge in this regard is still limited. Here we evaluated the molecular pathways modulated by cannabidiol compared to the antipsychotics clozapine (atypical) and haloperidol (typical), additionally evaluating the effects of benztropine, a muscarinic receptor antagonist that displays a protective effect in oligodendrocytes and myelination. For this purpose, we employed nano-chromatography coupled with mass spectrometry to investigate the proteomic response to these drugs both in healthy oligodendrocytic cells and in a cuprizone-based toxicity model, using the human oligodendrocyte precursor cell line MO3.13. Cannabidiol shares similarities of biochemical pathways with clozapine and benztropine, in agreement with other studies that indicated an atypical antipsychotic profile. All drugs tested affected metabolic and gene expression pathways and cannabidiol, benztropine, and clozapine modulated cell proliferation and apoptosis when administered after cuprizoneinduced toxicity. These general pathways are associated with cuprizone-induced cytotoxicity in MO3.13 cells, indicating a possible proteomic approach when acting against the toxic effects of cuprizone. In conclusion, although modeling oligodendrocytic cytotoxicity with cuprizone does not represent the entirety of the pathophysiology of oligodendrocyte impairments, these results provide insight into the mechanisms associated with the effects of cannabidiol and antipsychotics against cuprizone toxicity, offering new directions of study for myelin-related processes and deficits. 


\section{INTRODUCTION}

Schizophrenia is a severe and chronic mental disorder that affects over 20 million people worldwide. It is characterized by positive, negative, and cognitive symptoms (Azorin et al., 2014; Owen et al., 2016) which are primarily treated with antipsychotics. These are classified as typical, such as haloperidol, or atypical, including clozapine, the prototype of this drug class (Leucht et al., 2009; van Os and Kapur, 2009) and has since become an indispensable part of treatment in the clinic. Recently, cannabidiol-a compound of Cannabis sativa-has been proposed as a new treatment for schizophrenia, since it has been shown to decrease positive symptoms and improve cognitive performance (Leweke et al., 2012; McGuire et al., 2018). Cannabidiol appears to present a pharmacological profile similar to atypical antipsychotics (Gururajan et al., 2011; Zuardi et al., 2012); however, its effects on oligodendrocytes (OLs) are still to be better understood.

OLs are cells present in brain white matter and are responsible for axon myelination, and dysfunction of this process has been implicated in neuronal dysconnectivity (Cassoli et al., 2015; Jørgensen et al., 2016; Vikhreva et al., 2016). This dysconnectivity and abnormalities in white matter have been implicated in the pathophysiology of schizophrenia (Bernard et al., 2015; Orban et al., 2017; Saito et al., 2018). Impairments in oligodendrogenesis and differentiation of oligodendrocyte progenitor cells (OPCs) have also been documented in schizophrenia (Hattori et al., 2014; Santos et al., 2019). Several findings suggest that antipsychotics and cannabidiol affect OLs and myelination, indicating a potential therapeutic effect in schizophrenia and neurodegenerative disorders (Bartzokis et al., 2007, 2009; Zajicek and Apostu, 2011; Rahimi et al., 2015). Thus, the biology of OPCs and mature OLs is an essential focal point to understand the role of these cells in the pathophysiology of-and the development of new treatment for-schizophrenia (de Almeida and Martins-deSouza, 2018).

Several models exist at various stages of development to comprehend the mechanisms of myelination, demyelination, and remyelination, including the in vitro and in vivo cuprizone demyelination models (Bénardais et al., 2013; Sachs et al., 2014; Xu et al., 2014, 2019; Taraboletti et al., 2017; de Rosa et al., 2019; Zahednasab et al., 2019). Cuprizone is a copperchelating agent that inhibits several enzymes that use copper as a cofactor, including cytochrome oxidase, succinyl dehydrogenase, and monoamine oxidase (Venturini, 1973; Petronilli and Zoratti, 1990; Messori et al., 2007). Consequently, this drug induces OL apoptosis, microglial activation, and demyelination in animals (Goldberg et al., 2013; Sachs et al., 2014). Disturbances in energy and protein metabolism have been reported as the molecular mechanisms of cuprizone toxicity, in addition to the resultant increases in oxidative and endoplasmic reticulum stress in OLs (Biancotti et al., 2008; Goldberg et al., 2013; Praet et al., 2014; Taraboletti et al., 2017). Conversely, benztropine is a molecule that enhances OL differentiation and myelination both in vitro and in vivo (Deshmukh et al., 2013; Ettle et al., 2016; Thompson et al., 2018). This compound is understood to act on immature OLs via muscarinic receptor antagonism (Deshmukh et al.,
2013; Ettle et al., 2016). However, these receptors' role and the molecular mechanisms behind the improvements in myelination and OL differentiation are unclear.

Therefore, we hypothesized the ability of cannabidiol to induce protective molecular mechanisms, with similarity to antipsychotics and benztropine in an in vitro cuprizone model. Proteomic changes induced by antipsychotics (haloperidol and clozapine), cannabidiol, and benztropine were quantified in oligodendrocytic cells (MO3.13) previously exposed to cuprizone. This study ascertained the degree to which cannabidiol and antipsychotics induce protective molecular mechanisms against cuprizone-mediated cytotoxicity in MO3.13 cells, subsequently comparing these results with benztropine.

\section{MATERIALS AND METHODS}

\section{Cell Culture and Treatment}

MO3.13 is an immortal oligodendrocytic human-human hybrid cell line that expresses phenotypic characteristics of immature OLs (Buntinx et al., 2003; Iwata et al., 2013), and has been previously used to study oligodendrocyte-like features (Iwata et al., 2013; Brandão-Teles et al., 2017; Jinsmaa et al., 2020). Here, MO3.13 cells were cultured in DMEM (Sigma-Aldrich, St. Louis, MO, United States) supplemented with $10 \%$ FBS and penicillin $100 \mathrm{IU} / \mathrm{ml}$, streptomycin $100 \mu \mathrm{g} / \mathrm{ml}$ and grown at $37^{\circ} \mathrm{C}$ and $5 \%$ $\mathrm{CO}_{2}$ as described previously (Iwata et al., 2013; Brandão-Teles et al., 2017).

Stocks of cuprizone (28.05 mM; Sigma-Aldrich, St. Louis, MO, United States), haloperidol (53 mM; Cristália), clozapine (25 mM; Cristália), or cannabidiol (25 mM; 99.6\% pure with no other cannabinoid, BSPG-Pharm, Sandwich) were prepared in DMSO, and the final concentration of DMSO in the medium was limited to $0.042 \%$. Benztropine $(6 \mathrm{mM})$ was dissolved in $\mathrm{ddH}_{2} \mathrm{O}$. Saline in DMSO (final concentration of DMSO in medium $0.042 \%$ ) was used as a control. Based on our pilot experiments and previous studies (Mecha et al., 2012; Bénardais et al., 2013; Deshmukh et al., 2013; Xu et al., 2014; Ettle et al., 2016), MO3.13 cells were seeded in six-well plates at a density of 115,000 cells/well, deprived of FBS overnight, and treated either with $10 \mu \mathrm{M}$ of cuprizone for $48 \mathrm{~h}$ or with $1 \mu \mathrm{M}$ haloperidol, clozapine, cannabidiol, or benztropine for $24 \mathrm{~h}$. For co-treatment conditions, cells were treated with cuprizone for a total of $48 \mathrm{~h}$; the co-treatment was added during the last $24 \mathrm{~h}$ of the treatment, all at the previously mentioned concentrations.

\section{MTT Assay}

For MTT colorimetric assays, cells were seeded in 96-well plates at a density of 7,000 cells/well for $24 \mathrm{~h}$ and deprived of FBS overnight. After incubation, the medium was aspirated and replaced with new media containing increasing cuprizone concentrations for $48 \mathrm{~h}$, or haloperidol, clozapine, cannabidiol, or benztropine for $8 \mathrm{~h}$. Subsequently, $0.5 \mathrm{mg} / \mathrm{ml}$ of MTT was added to each well and incubated for $2 \mathrm{~h}$ at $37^{\circ} \mathrm{C}$; ethanol $100 \%$ was added, and the absorbance was read at $570 \mathrm{~nm}$ on a plate spectrophotometer. Data are expressed as mean \pm SEM, and one-way analysis of variance (ANOVA) was used to analyze 
the statistical significance, followed by Dunnett's post-hoc test $(p<0.05)$.

\section{Proteome Extraction}

After treatment, cells were collected with a scraper and PBS (phosphate-buffered saline; final concentration $1 \mathrm{x}$ ), and centrifuged at $4{ }^{\circ} \mathrm{C}$ and $1,200 \times g$ for $5 \mathrm{~min}$. The pellets were homogenized in a lysis buffer (Tris pH 6.8, 1 M; SDS 20\%) with protease inhibitor (final working concentration, 1X; Roche; Mannheim, Germany). Each sample underwent a short run through a polyacrylamide gel to remove buffer salts and other contaminants before peptide digestion was performed in gel. One-hunderd microliters of $10 \mathrm{mM}$ dithiothreitol stock (15.4 mg DTT in $10 \mathrm{ml}$ of $50 \mathrm{mM}$ ammonium bicarbonate-Ambic), $100 \mu \mathrm{l}$ of $55 \mathrm{mM}$ iodoacetamide stock (102 mg IAA in $10 \mathrm{ml}$ of $50 \mathrm{mM}$ Ambic), and $200 \mu \mathrm{l}$ acetonitrile $100 \%$ were added in each sample. Proteins were digested by trypsin for $16-18 \mathrm{~h}$ at $37^{\circ} \mathrm{C}$ and the peptide concentrations were determined by the BSA assay kit (Sigma-Aldrich, St. Louis, MO, United States).

\section{Tandem Mass Spectrometry}

Proteomic samples were separated two-dimensionally and analyzed by a nanoAcquity UPLC M-Class (Waters Corporation, Milford, MA, USA) liquid chromatograph coupled to a Synapt G2-Si mass spectrometer (Waters Corporation, Milford, MA, USA). Each sample was fractionated into five fractions in the first dimension using discontinuous acetonitrile steps $(11.4,14.7$, $17.4,20.7$, and $50 \%$ ) before separation in the second dimension with a continuous acetonitrile gradient from $7 \%$ to $40 \%(\mathrm{v} / \mathrm{v})$ on C18 columns. Peptides were ionized by electrospray ionization in positive mode and [Glu 1]-Fibrinopeptide B was used as the lock-mass. MS/MS analyses were performed in data-independent acquisition (DIA) mode using $\mathrm{UDMS}^{\mathrm{E}}$ after manually creating an ion selection method profile for each sample fraction using DriftScape (version 2.9).

\section{Data Processing and Protein Identification}

MS/MS spectra were aligned and analyzed with Progenesis ${ }^{\circledR}$ QI for Proteomics (version 3.1) with Apex3D, peptide 3D, and ion accounting informatics (Waters). Proteins were identified using the reviewed Uniprot Homo sapiens database (obtained 12/2020) and quantified using the default Top3 method. The following parameters were used in the identification of peptides/proteins: digestion by trypsin with a maximum of one missed cleavage; maximum protein mass of $600 \mathrm{kDa}$; false discovery rate (FDR) less than $1 \%$ (as calculated by a reverse sequence database generated on-the-fly by Progenesis); at least two fragments per peptide, one peptide per protein, and five or more fragments per protein; at least one unique peptide per protein for quantitation; and a mass error $\leq 20 \mathrm{ppm}$. Proteins were considered differentially expressed when the one-way analysis of variance (ANOVA) returned a $p$-value $<0.05$.

\section{In silico Analyses}

Using proteins considered to be differentially expressed, affected biological processes and biochemical pathways were analyzed using Reactome (Fabregat et al., 2018), David bioinformatics database (Huang et al., 2009a,b), and Proteomaps
(Liebermeister et al., 2014). Biological processes and protein networks were analyzed using Metascape (Zhou et al., 2019), Cytoscape version 3.8.2 (Bindea et al., 2009; Lotia et al., 2013; Franz et al., 2016; Szklarczyk et al., 2017), and ClustVis (Metsalu and Vilo, 2015).

\section{RESULTS}

\section{MTT Assay}

Cuprizone-induced toxicity was measured in the human oligodendrocytic cell line MO3.13 using increasing cuprizone concentrations for $48 \mathrm{~h}$ via MTT assay. All cells treated with cuprizone displayed a loss of viability after $48 \mathrm{~h}$ (Supplementary Figure 1A). The concentrations used in previous studies for these antipsychotics, cannabidiol, and benztropine (Mecha et al., 2012; Deshmukh et al., 2013; Xu et al., 2014) were confirmed to not be cytotoxic to the MO3.13 cell line under this experiment's conditions. This procedure was achieved by MTT assay, confirming that the cytotoxic cutoff for these compounds at $8 \mathrm{~h}$ of incubation is sufficiently above these concentrations. Viability dropped to a significant degree after $75 \mu \mathrm{M}$ clozapine, $50 \mu \mathrm{M}$ haloperidol, $4 \mu \mathrm{M}$ cannabidiol, and $100 \mu \mathrm{M}$ benztropine (Supplementary Figures 1B-E). The previously published concentration of $10 \mu \mathrm{M}$ cuprizone induced a cytotoxic effect in these cells and concentrations of $1 \mu \mathrm{M}$ for antipsychotics, cannabidiol, and benztropine (Mecha et al., 2012; Bénardais et al., 2013; Deshmukh et al., 2013; Xu et al., 2014; Taraboletti et al., 2017) were not cytotoxic and therefore used during treatment, and co-treatment assays.

\section{Proteomic Analyses}

\section{Proteomic Signature of MO3.13 Cells}

Although the MO3.13 cell line does not exhibit the main features of myelinating OLs nor those of late-stage OPCs that could differentiate into mature OLs, these cells can be useful for the investigations of early stages of OPCs. We previously showed that the MO3.13 cell line submitted to a differentiation protocol presents an increase in the marker PLP, suggesting its OPC-like features (Seabra et al., 2019, 2020). Here, we compared the whole proteome identified in our experiments with the databanks for OLs and OPCs (Zhang et al., 2019), whereupon we found several protein markers in our data, including RAB2A, TUBB, NPM1, LDHB, MAP1A, NACA, DHCR7, TMEFF2, BIN1, NAP1L1, MARCKSL1, EIF3E, DHCR24, RPS2, LIMA1, GSN, SCD, EEF1B2, TUBB3, EEF2, PGRMC1, NME2, ACAT2, MAP2, EIF3L, RPL13A, SNX1, CNP, MIF, HIP1, FDPS, GAP43, CDK18, PDGFRA, MICAL1, RPL31, CDH13, RPS23, TM7SF2, and TUBB4A. Together, these data reinforce that MO3.13 cells can be a useful approach to investigate the molecular pathways for an early stage of OPCs. In line with these data, other studies have also shown oligodendrocytic markers in MO3.13 cells (McLaurin et al., 1995; Buntinx et al., 2003).

\section{Cannabidiol, Antipsychotics, and Benztropine Individually}

A total of 1,890 proteins were identified and quantified. When compared to vehicle, differentially expressed proteins were 
found in each condition: cannabidiol, 93; haloperidol, 97; clozapine, 278; and benztropine, 191 (see Supplementary Table 1). The most extensive overlap of proteins and pathways affected in the same direction was observed between clozapine and benztropine, while cannabidiol shared a higher overlap of pathways with clozapine and benztropine than with haloperidol (Figures 1A,B,D). Regarding biological processes, all drugs caused changes in RNA metabolism, cytoplasmic translation, ncRNA metabolic process, actin cytoskeleton organization, heterocycle catabolic process, generation of precursor metabolites and energy, ribonucleoprotein complex biogenesis, and RNA localization (Figure 1B). Treatment with benztropine, cannabidiol, or antipsychotics additionally modulated proteins related to various metabolic processes (Figures 1C,D and Supplementary Figures 2B-E) and antioxidant defense (Supplementary Table 2).

\section{Proteome of MO3.13 Cells Treated With Cuprizone}

A total of 1,889 proteins were identified and quantified, 44 of which were differentially expressed in MO3.13 cells in response to cuprizone (see Supplementary Table 1). In line with the literature (Werner et al., 2010; Taraboletti et al., 2017; Szilagyi et al., 2020) here we also identified proteins related to metabolism, including L-lactate dehydrogenase A chain (LDHA), polyunsaturated fatty acid 5-lipoxygenase (ALOX5), and dihydrolipoyl lysine-residue succinyltransferase component of 2-oxoglutarate dehydrogenase (DLST; Supplementary Table 2). Cuprizone was also found to modulate proteins related to amino acid metabolism and genetic processes, including translational, spliceosome processes (FDR: 0.0064, String-DB), and RNA splicing (Figure 2 and Supplementary Figure 2A).

\section{Proteomic Changes in Co-treated MO3.13 Cells}

A total of 1890 and 1889 proteins were identified and quantified for CPZCBD and CPZCLZ and CPZHAL and CPZBENZ, respectively. When compared to cells insulted with cuprizone, differentially expressed proteins were found in co-treatment with cannabidiol (CPZCBD, 89), haloperidol (CPZHAL, 92), clozapine (CPZCLZ, 75), and benztropine (CPZBENZ, 150; see Supplementary Table 1). Unlike the pathway clustering organization observed when drugs were administered alone (Figure 1B), the comparison of co-treatments indicated that cannabidiol shares more proteins that are dysregulated in the same direction with benztropine than with haloperidol or clozapine (Figures 4A,B). All co-treatments caused changes [ $-\log _{10}(\mathrm{P})$ from 20 to 2] in proteins related to RNA metabolism and RNA splicing (Figures 3, 4B). For details regarding proteins and genes, see Figure 4B and Supplementary Figure 3. Moreover, these co-treatments modulated proteins seen in cuprizone insult, including protein disulfide-isomerase, heterogeneous nuclear ribonucleoprotein $\mathrm{K}$, T-complex protein 1 subunit zeta, and protein LYRIC, which were all modulated in both cannabidiol and benztropine co-treatments, with both antipsychotics also modulating myeloid-associated differentiation marker (MYADM).

\section{DISCUSSION}

Comparing the effects of cannabidiol and antipsychotics with the putative protective function of benztropine on the MO3.13 proteome, we found that all drugs modulated proteins related to metabolism, antioxidant system, and RNA splicing. Here, we found changes in the constituent proteins of several molecular mechanisms and biological processes in oligodendrocytic cells resulting from benztropine administration, which might be associated with its ability to induce myelination and OPC differentiation. We then compared these results with MO3.13 cells treated with cannabidiol and two antipsychotics (haloperidol and clozapine) in terms of their hypothesized ability to induce protective molecular mechanisms.

First, we showed that benztropine modulated proteins related to energy, cholesterol, and fatty acid metabolism, which might be associated with its mechanisms of inducing cholesterol biogenesis in OLs and myelination (Ettle et al., 2016; Hubler et al., 2018). Cannabidiol and antipsychotics are previously known to modulate proteins related to catabolic processes. Studies have demonstrated that cannabidiol can attenuate cell damage by promoting mitochondrial function and biogenesis, thus modulating glucose metabolism in neurons (Sun et al., 2017). The observation that cannabidiol also alters metabolic pathways in MO3.13 cells supports the previous studies and helps validate these findings. Clozapine treatment also modulated proteins related to energy and lipid metabolism. Regarding haloperidol, its administration has been associated with changes in proteins related to carbohydrates and lipid metabolism (Brandão-Teles et al., 2019), similar to our results; however, it inhibits cholesterol biosynthesis in cell culture (Sánchez-Wandelmer et al., 2010). The protective effects of these drugs on MO3.13 cells can be attributed to a modulation of metabolism, similar to benztropine. The result that benztropine modulates proteins related to energy metabolism, as cannabidiol, haloperidol, and clozapine do, suggests that this may be a shared mechanism of action for protective effects.

Our results also reported that benztropine modulated a protein related to antioxidant defense. The antioxidant effects of benztropine are well known in neuronal cells (Cerles et al., 2019). Nonetheless, we found that benztropine downregulated disulfide-isomerase A3, which attenuates oxidative and endoplasmic reticulum stress (Yoo et al., 2019). We found that clozapine modulated the protein superoxide dismutase, related to antioxidant defense, and both cannabidiol and haloperidol modulated proteins related to selenocysteine synthesis and selenoamino acid metabolism. While haloperidol has been suggested to induce oxidative stress in rat brains (Andreazza et al., 2015), clozapine has been described to promote an antioxidant effect (Hendouei et al., 2018). Similarly, cannabidiol has been observed to prevent hydrogen peroxideinduced apoptosis in nucleus pulposus cells and decrease oxidative species in OPCs (Mecha et al., 2012; Chen et al., 2016), suggesting a possible cause of the differences in the side effect profiles of antipsychotics and cannabidiol.

After comparing the individual effects of cannabidiol, antipsychotics, and benztropine, we investigated these drugs' 


\section{A}

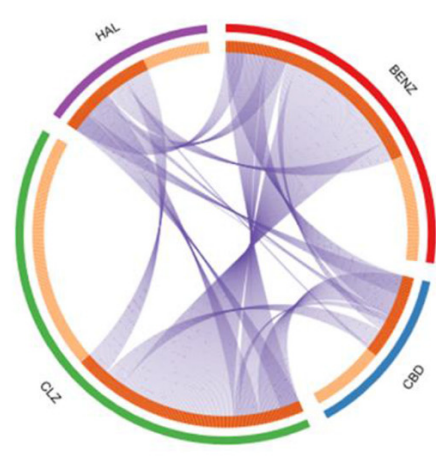

B

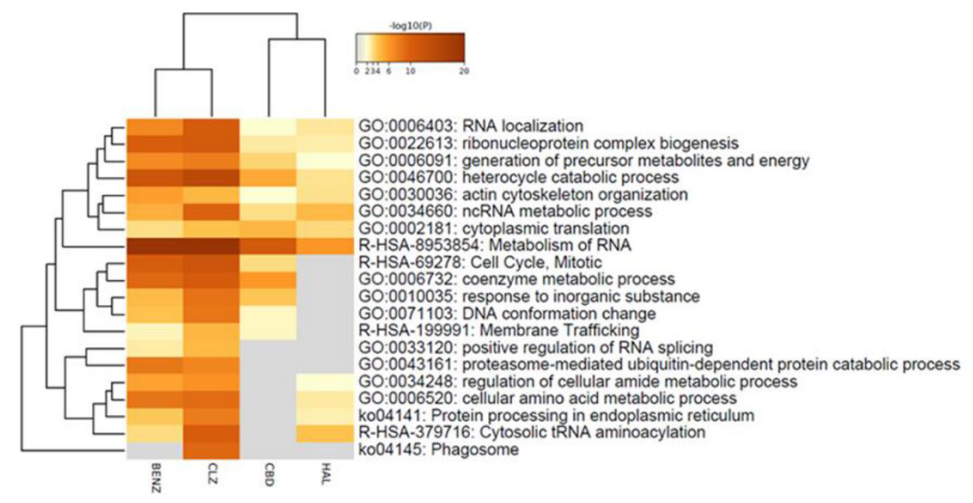

C

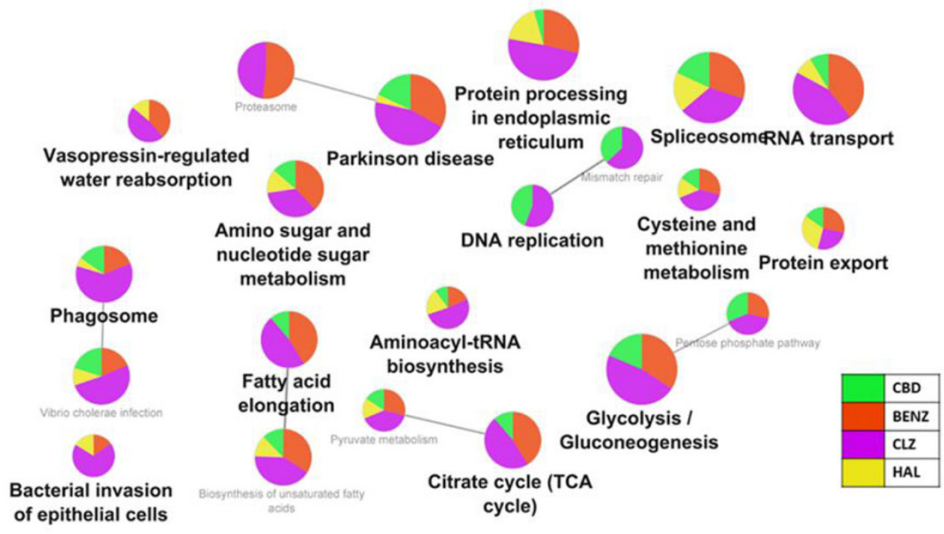

D

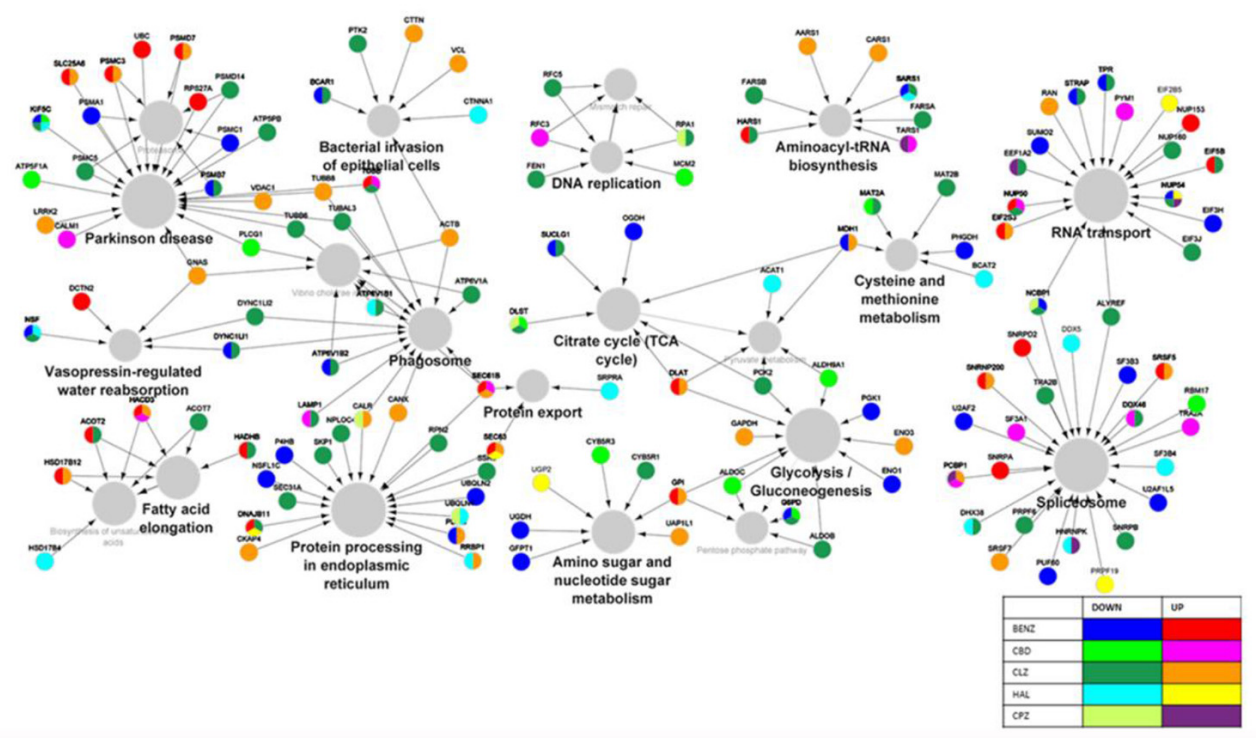

FIGURE 1 | (A) Chord diagram. (B) Heatmap of enriched pathways (Metascape). (C) Network of pathways affected by cannabidiol (CBD), benztropine (BENZ), clozapine (CLZ), and haloperidol (HAL) treatments, according to proteomic analysis, showing the percentage of visible genes of each term or pathway. (D) Network of pathways and proteins affected by cannabidiol, benztropine, clozapine, and haloperidol treatments, and cuprizone (CPZ) insult, according to proteomic analysis, showing hub genes that are shared in the enriched KEGG pathways. The gene ontology interaction network was obtained by functional enrichment analysis using the ClueGO plug-in in Cytoscape. All terms were selected by significance $(p<0.05)$ following a Benjamini-Hochberg correction. 


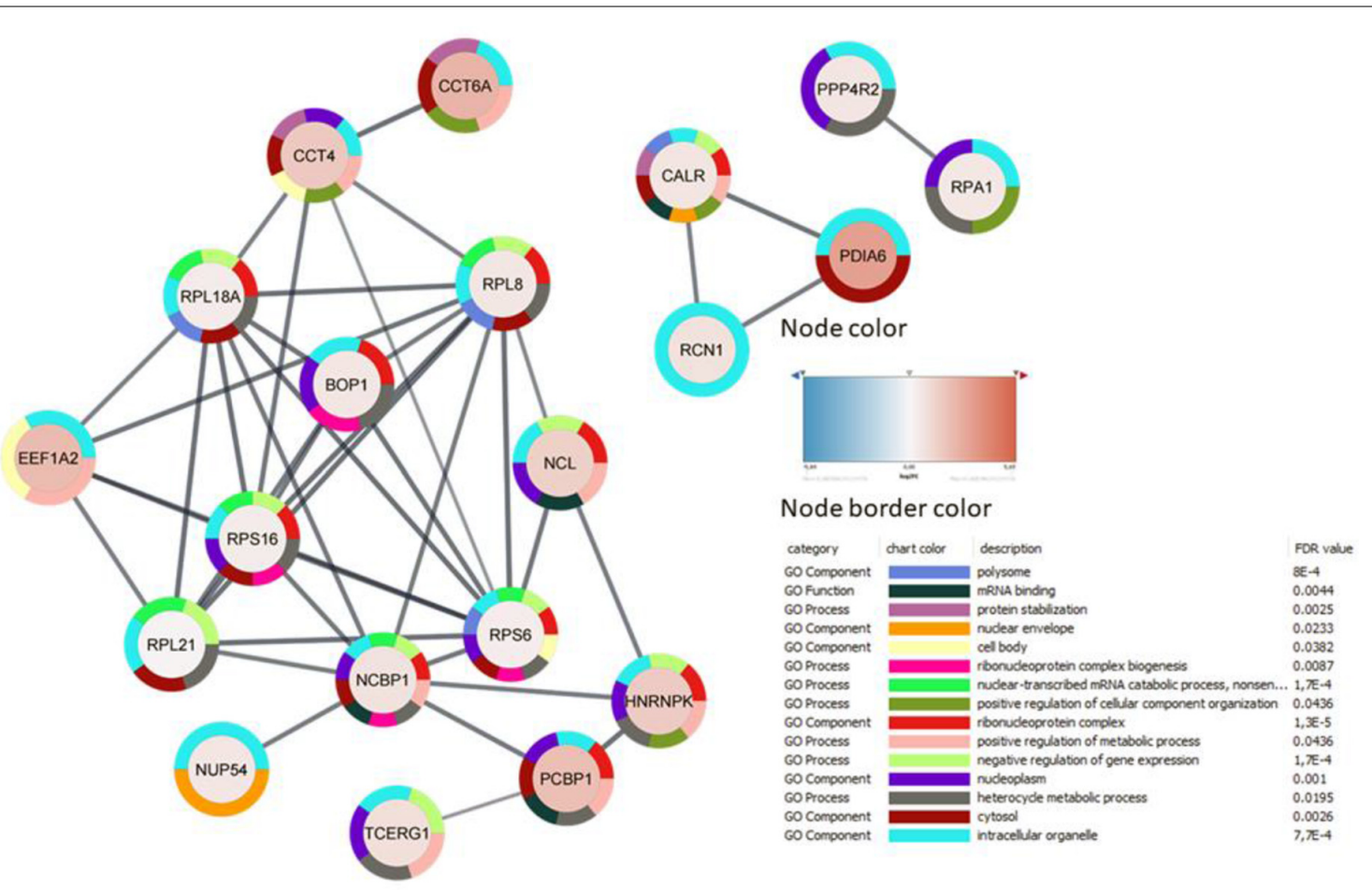

FIGURE 2 | Protein-protein interaction of 44 genes affected by cuprizone treatment, analyzed with STRING in Cytoscape. Proteins without interactions within the network were omitted. Nodes represent proteins, their colors indicate the fold-change in the expressed pattern after cuprizone treatment, and lines represent interactions between them. Node border colors indicate the top 15 gene ontology terms.

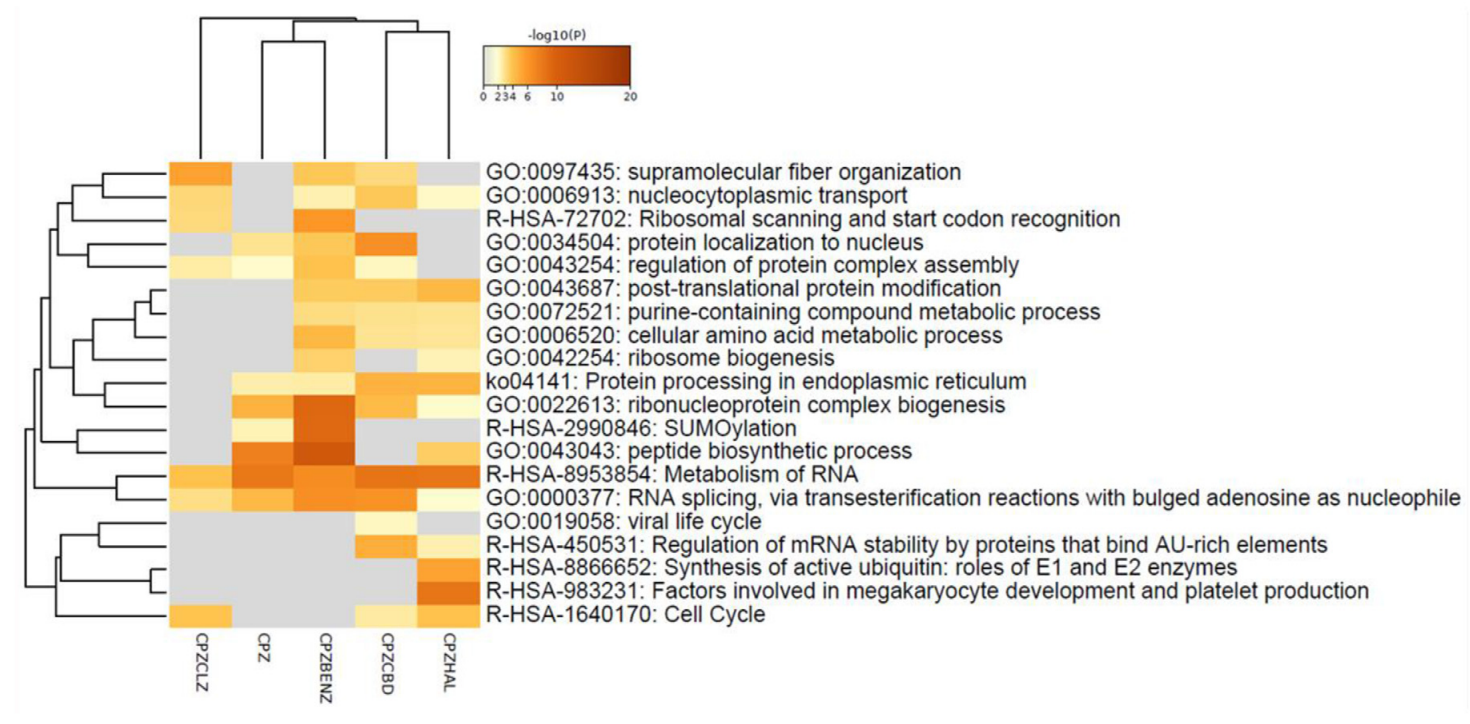

FIGURE 3 | Heatmap of enriched pathways of MO3.13 cells insulted with cuprizone (CPZ) or co-treated with cuprizone and haloperidol (CPZHAL), cannabidiol (CPZCBD), clozapine (CPZCLZ), or benztropine (CPZBENZ). Created in Metascape.

molecular mechanisms against cuprizone-mediated toxicity in MO3.13 cells. Cuprizone insult resulted in changes in proteins related to metabolism and genetic processes, both of which were also modulated by benztropine treatment. More specifically, benztropine increased some proteins related to fatty acid elongation and decreased proteins related to energy metabolism, as well as both up-and down-regulating proteins related to the spliceosome and RNA transport (Figure 1D). 


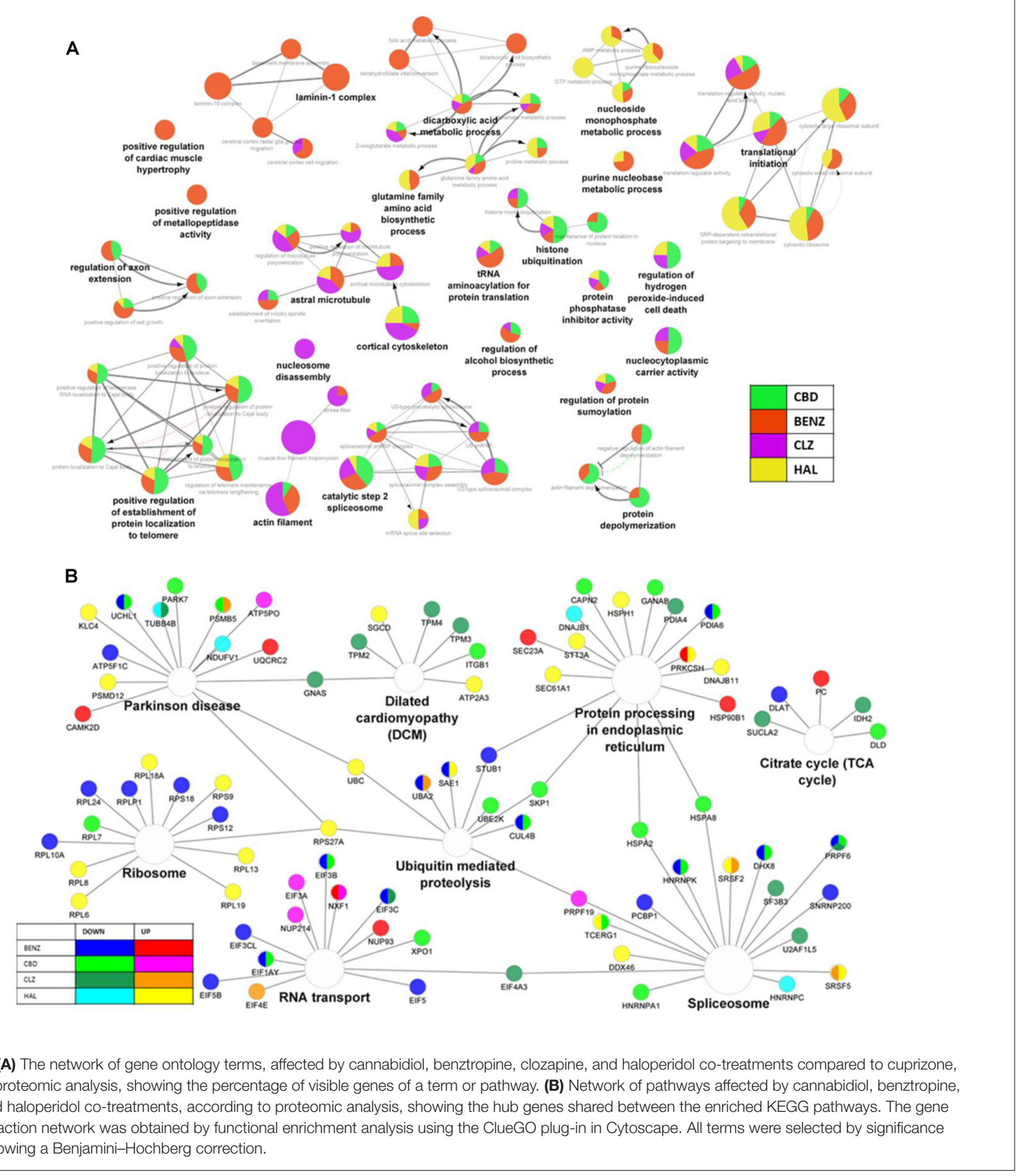

FIGURE 4 | (A) The network of gene ontology terms, affected by cannabidiol, benztropine, clozapine, and haloperidol co-treatments compared to cuprizone, according to proteomic analysis, showing the percentage of visible genes of a term or pathway. (B) Network of pathways affected by cannabidiol, benztropine, clozapine, and haloperidol co-treatments, according to proteomic analysis, showing the hub genes shared between the enriched KEGG pathways. The gene ontology interaction network was obtained by functional enrichment analysis using the ClueGO plug-in in Cytoscape. All terms were selected by significance $(p<0.05)$ following a Benjamini-Hochberg correction.

We identified bidirectional modulations in apoptosis and cell proliferation proteins in cannabidiol, clozapine, and haloperidol co-treatments, which were mostly downregulated in response to cuprizone insult. Cannabidiol and benztropine co-treatments increased heterogeneous nuclear ribonucleoprotein $\mathrm{K}$ after cuprizone-induced downregulation, a protein which regulates proliferation and apoptosis (Chen et al., 2017). Cannabidiol, benztropine, and clozapine co-treatments also decreased the protein LYRIC, an activator of the transcription factor
NF- $\kappa \mathrm{B}$ that affects neuronal plasticity, neurodegeneration, and neuronal development (O'Neill and Kaltschmidt, 1997) and which was found increased after cuprizone insult. Lastly, clozapine increased DnaJ homolog subfamily A member 3, a modulator of apoptotic signal transduction that was decreased after cuprizone insult. Though there is significant overlap between clozapine, benztropine, and cannabidiol (Figures 1, 3, 4), each compound seems to affect OL proliferation and apoptosis in distinct ways; these compounds 
must be tested further regarding their potential to promote cell proliferation.

Regarding energy and lipid metabolism, cuprizone decreases glucose utilization (Taraboletti et al., 2017; Kim et al., 2019), affects NAD+ metabolism, and blocks glycolytic pathways (Taraboletti et al., 2017). In our study, cuprizone appeared to affect metabolic proteins and may impair ATP production, and affect the antioxidant system. Benztropine co-treatment induced changes in metabolic processes, in which modulated proteins were most specifically related to energy and amino acid metabolism, additionally downregulating proteins related to lipid metabolism (Figure 4B). These data, therefore, indicate benztropine's potential to attenuate the metabolic disturbances seen in the cuprizone model. All co-treatments modulated varying proteins involved in energy and lipid metabolism, and in particular, all drugs increased the expression of aspartate aminotransferase, related to NAD redox balance, which was altered during cuprizone administration (Taraboletti et al., 2017). However, disulfide-isomerase A6, a redox activity regulator which was upregulated in cuprizone insult, was downregulated only by cannabidiol and benztropine cotreatments. Cannabidiol co-treatment modulated dihydrolipoyl dehydrogenase and ATP synthase subunit $\mathrm{O}$, which are related to ATP production. Haloperidol co-treatment also modulated proteins related to energy metabolism, including ATP-dependent 6-phosphofructokinase and $\mathrm{NADH}$ dehydrogenase flavoprotein 1. Since clozapine did not significantly modulate biological pathways deregulated by cuprizone, it is interesting that in an in vivo cuprizone model, clozapine treatment could not prevent demyelination during cuprizone administration; however, it did enhance functional support after demyelination (Templeton et al., 2019). Here, we showed that clozapine instead modulates some critical proteins related to energy metabolism, such as succinate-CoA ligase and isocitrate dehydrogenase. Thus, we demonstrated that antipsychotics modulate proteins related to metabolism and, similarly, cannabidiol and benztropine modulate this pathway and the antioxidant system throughout cuprizone insult, suggesting a potential ability to prevent demyelination events.

Last, our data also show that cuprizone modulated proteins related to amino acid metabolism and genetic processes, including translation and spliceosome processes. Amino acid perturbations and deregulations in the antioxidant system have already been documented in mice exposed to cuprizone (Goldberg et al., 2013; Taraboletti et al., 2017). Our results agree with the hypothesis that cuprizone can affect amino acid metabolism, including selenoamino acid metabolism. All co-treatments downregulated some proteins related to spliceosomes and RNA metabolism (Figure 4B); and post-translational modification processes were modulated by benztropine, cannabidiol, and haloperidol. In particular, RNA splicing pathways, downregulated after cuprizone insult, showed higher expression of some constituent proteins during the antipsychotic co-treatments (Figures 1C, 4B). Benztropine, however, decreased poly ( $\mathrm{rC}$ )-binding protein 1 , which also participates in RNA splicing, and cannabidiol decreased the levels of threonine-tRNA ligase 1 , a protein that participates in protein biosynthesis. It is worth noting that both cannabidiol and benztropine decreased heterogeneous nuclear ribonucleoprotein $\mathrm{K}$, which is a protein related to spliceosome function that was found upregulated after cuprizone insult. Though each co-treatment affected these pathways differently, a more developed understanding of how these pathways are affected by cannabidiol, antipsychotics, and benztropine is essential to help determine how OLs regenerate after toxic effects like those induced by cuprizone, and how schizophrenia and other demyelinating disorders can be better treated.

This study, of course, has its limitations. First, the MO3.13 cell line is not able to properly model myelination and remyelination processes since the cells do not differentiate into myelinating or mature OLs and are not able to myelinate axons (De Kleijn et al., 2019). Second, the cell line is not suitable as a model for the multifactorial disease since it does not carry any genotypic or phenotypic features of patients. Nonetheless, this oligodendrocytic cell line has been previously used to study the early stages of OPC features (Iwata et al., 2013; Brandão-Teles et al., 2017; Jinsmaa et al., 2020) and we previously showed that MO3.13 cells submitted to a short maturation protocol exhibit an increase in PLP, highlighting the utility of these cells to investigate molecular pathways related to the early stages of OPCs (Seabra et al., 2019, 2020). Third, the proteomic analyses performed herein point to several altered pathways and biological processes; but a validation of these findings using other approaches is fundamental to confirm the effects on the functionality of these, and other, cells. In conclusion, our study provides evidence that cannabidiol can modulate signaling pathways similarly to clozapine and benztropine, depicting the biochemical mechanisms behind such modulations. After the cuprizone-induced insult, cannabidiol exhibited similarities with benztropine, clozapine, and haloperidol. Cannabidiol and antipsychotics mainly affect metabolism, cell proliferation, apoptosis, cell redox-homeostasis, and genetic factors. There is an overlap in the pathways affected by cannabidiol, benztropine, and antipsychotics. Since this overlap includes pathways that are documented to be related to protective effects in OLs, we suggest that cannabidiol is indeed a potential protective compound against cuprizone insult in oligodendrocytic cells, which might be related to the demyelination process, a hypothesis that must be confirmed using in vivo and more complex in vitro models.

\section{DATA AVAILABILITY STATEMENT}

The mass spectrometry proteomics data have been deposited to the ProteomeXchange Consortium via the PRIDE partner repository with the dataset identifier PXD028419.

\section{AUTHOR CONTRIBUTIONS}

ACBF conducted the experimental part, analyzed data, and wrote the manuscript. BS helped with data analysis, manuscript revision, and English review. LS-C helped with data analysis and figures. AV and FC performed validation experiments. DM-d-S supervised the whole process and provided funding. DM-d-S and VA designed and conceived the study and edited the final version 
of the manuscript. $\mathrm{AZ}, \mathrm{JH}$, and JC provided cannabidiol for experiments, and contributed to manuscript revision. All authors contributed to the article and approved the submitted version.

\section{FUNDING}

This work was supported by the São Paulo Research Foundation (Fundação de Amparo à Pesquisa do Estado de São Paulo, FAPESP, grant numbers 2017/25588-1, 2017/18242-1, 2018/03673-0, 2018/10362-0, 2018/03422-7, 2018/03450$0,2019 / 22398-2,2019 / 00098-7)$, the Coordination for the Improvement of Higher Education Personnel (Coordenação de Aperfeiçoamento de Pessoal de Nível Superior, CAPES, grant number 88887.495565/2020-00), and the CNPq (Conselho Nacional de Desenvolvimento Científico e Tecnológico, grant number 157265/2018-8, 166571/2018-0). This work was also supported by the Instituto Nacional de Ciência e Tecnologia Translational em Medicina (INCT-TM; CNPq/FAPESP; 2008/09009-2); JC received a grant from the University Global Partnership Network (UGPN) -Global Priorities in Cannabinoid Research Excellence Program. DM-d-S, JC, JH, and $\mathrm{AZ}$ are recipients of $\mathrm{CNPq}$ research fellowships.

\section{ACKNOWLEDGMENTS}

We thank FAPESP, CNPq, and CAPES for financial support. We thank Paulo Baldasso and Prof. Dr. Marcelo Bispo de Jesus for the support.

\section{REFERENCES}

Andreazza, A. C., Barakauskas, V. E., Fazeli, S., Feresten, A., Shao, L., Wei, V., et al. (2015). Effects of haloperidol and clozapine administration on oxidative stress in rat brain, liver and serum. Neurosci. Lett. 591, 36-40. doi: 10.1016/j.neulet. 2015.02.028

Azorin, J.-M., Belzeaux, R., and Adida, M. (2014). Negative symptoms in schizophrenia: where we have been and where we are heading. CNS Neurosci. Ther. 20, 801-808. doi: 10.1111/cns.12292

Bartzokis, G., Lu, P. H., Nuechterlein, K. H., Gitlin, M., Doi, C., Edwards, N., et al. (2007). Differential effects of typical and atypical antipsychotics on brain myelination in schizophrenia. Schizophr. Res. 93, 13-22. doi: 10.1016/j.schres. 2007.02.011

Bartzokis, G., Lu, P. H., Stewart, S. B., Oluwadara, B., Lucas, A. J., Pantages, J., et al. (2009). In vivo evidence of differential impact of typical and atypical antipsychotics on intracortical myelin in adults with schizophrenia. Schizophr. Res. 113, 322-331. doi: 10.1016/j.schres.2009.06.014

Bénardais, K., Kotsiari, A., Skuljec, J., Koutsoudaki, P. N., Gudi, V., Singh, V., et al. (2013). Cuprizone [bis(cyclohexylidenehydrazide)] is selectively toxic for mature oligodendrocytes. Neurotox. Res. 24, 244-250. doi: 10.1007/s12640013-9380-9

Bernard, J. A., Orr, J. M., and Mittal, V. A. (2015). Abnormal hippocampalthalamic white matter tract development and positive symptom course in individuals at ultra-high risk for psychosis. NPJ Schizophr. 1:15009. doi: 10.1038/npjschz.2015.9

Biancotti, J. C., Kumar, S., and de Vellis, J. (2008). Activation of inflammatory response by a combination of growth factors in cuprizone-induced demyelinated brain leads to myelin repair. Neurochem. Res. 33, 2615-2628. doi: 10.1007/s11064-008-9792-8

Bindea, G., Mlecnik, B., Hackl, H., Charoentong, P., Tosolini, M., Kirilovsky, A., et al. (2009). ClueGO: a Cytoscape plug-in to decipher functionally grouped gene ontology and pathway annotation networks. Bioinformatics 25, 1091-1093. doi: 10.1093/bioinformatics/btp101

\section{SUPPLEMENTARY MATERIAL}

The Supplementary Material for this article can be found online at: https://www.frontiersin.org/articles/10.3389/fnmol. 2021.673144/full\#supplementary-material.

SUPPLEMENTARY FIGURE 1 | A MTT assay was used to measure the percentage of live MO3.13 cells after $48 \mathrm{~h}$ and $8 \mathrm{~h}$ in growth medium (DMEM), Vehicle (for cuprizone: DMSO, 0.3\%; haloperidol and cannabidiol: DMSO, 0.3\%; clozapine: DMSO, 0.4\%; benztropine: water), and increasing concentrations of cuprizone for $48 \mathrm{~h}$ (A), haloperidol (B), clozapine (C), cannabidiol (D), or benztropine (E) for $8 \mathrm{~h}\left({ }^{*} P \leq 0.05\right.$; ${ }^{* * *} P<0.001$; ${ }^{* * * *} P<0.0001$; $n=6$ wells/condition).

SUPPLEMENTARY FIGURE 2 | Bar graph of enriched biochemical pathways in MO3.13 cells treated with cuprizone $10 \mu \mathrm{M}, 48 \mathrm{~h}$ (A), haloperidol (B), clozapine (C), cannabidiol (D), and benztropine (E), colored by $p$-value, created in Metascape. Colored lines represent $p$-values, which range between $10^{-20}$ and $10^{-2}$.

SUPPLEMENTARY FIGURE 3 | Network of pathways affected by cannabidiol, benztropine, clozapine, and haloperidol co-treatments, according to proteomic analysis, showing the percentage of visible genes of a term or pathway and the hub genes that enriched KEGG pathways. The gene ontology interaction network was obtained by functional enrichment analysis using the ClueGO plug-in in Cytoscape. All terms were selected by significance $(p<0.05)$ following a Benjamini-Hochberg correction.

SUPPLEMENTARY TABLE 1 | Proteins differentially regulated by treatments.

SUPPLEMENTARY TABLE 2 | Pathways associated with proteome changes induced by treatments.

Brandão-Teles, C., de Almeida, V., Cassoli, J. S., and Martins-deSouza, D. (2019). Biochemical pathways triggered by antipsychotics in human [corrected] oligodendrocytes: potential of discovering new treatment targets. Front. Pharmacol. 10:186. doi: 10.3389/fphar.2019. 00186

Brandão-Teles, C., Martins-de-Souza, D., Guest, P. C., and Cassoli, J. S. (2017). MK-801-treated oligodendrocytes as a cellular model to study schizophrenia. Adv. Exp. Med. Biol. 974, 269-277. doi: 10.1016/j.jaip.2021. 03.059

Buntinx, M., Vanderlocht, J., Hellings, N., Vandenabeele, F., Lambrichts, I., Raus, J., et al. (2003). Characterization of three human oligodendroglial cell lines as a model to study oligodendrocyte injury: morphology and oligodendrocyte-specific gene expression. J. Neurocytol. 32, 25-38. doi: 10.1023/a:1027324230923

Cassoli, J. S., Guest, P. C., Malchow, B., Schmitt, A., Falkai, P., and Martinsde-Souza, D. (2015). Disturbed macro-connectivity in schizophrenia linked to oligodendrocyte dysfunction: from structural findings to molecules. NPJ Schizophr. 1:15034. doi: 10.1038/npjschz.2015.34

Cerles, O., Gonçalves, T. C., Chouzenoux, S., Benoit, E., Schmitt, A., Bennett Saidu, N. E., et al. (2019). Preventive action of benztropine on platinuminduced peripheral neuropathies and tumor growth. Acta Neuropathol. Commun. 7:9. doi: 10.1186/s40478-019-0657-y

Chen, J., Hou, C., Chen, X., Wang, D., Yang, P., He, X., et al. (2016). Protective effect of cannabidiol on hydrogen peroxide-induced apoptosis, inflammation and oxidative stress in nucleus pulposus cells. Mol. Med. Rep. 14, 2321-2327. doi: $10.3892 / \mathrm{mmr} .2016 .5513$

Chen, X., Gu, P., Xie, R., Han, J., Liu, H., Wang, B., et al. (2017). Heterogeneous nuclear ribonucleoprotein $\mathrm{K}$ is associated with poor prognosis and regulates proliferation and apoptosis in bladder cancer. J. Cell. Mol. Med. 21, 1266-1279. doi: $10.1111 / \mathrm{jcmm} .12999$

de Almeida, V., and Martins-de-Souza, D. (2018). Cannabinoids and glial cells: possible mechanism to understand schizophrenia. Eur. Arch. Psychiatry Clin. Neurosci. 268, 727-737. doi: 10.1007/s00406-018-0874-6 
De Kleijn, K. M. A., Zuure, W. A., Peijnenborg, J., Heuvelmans, J. M., and Martens, G. J. M. (2019). Reappraisal of human HOG and MO3.13 cell lines as a model to study oligodendrocyte functioning. Cells 8:1096. doi: 10.3390/ cells 8091096

de Rosa, V., Secondo, A., Pannaccione, A., Ciccone, R., Formisano, L., Guida, N., et al. (2019). D-aspartate treatment attenuates myelin damage and stimulates myelin repair. EMBO Mol. Med. 11:e9278. doi: 10.15252/emmm.2018 09278

Deshmukh, V. A., Tardif, V., Lyssiotis, C. A., Green, C. C., Kerman, B., Kim, H. J., et al. (2013). A regenerative approach to the treatment of multiple sclerosis. Nature 502, 327-332. doi: 10.1038/nature12647

Ettle, B., Kerman, B. E., Valera, E., Gillmann, C., Schlachetzki, J. C. M., Reiprich, S., et al. (2016). $\alpha$-Synuclein-induced myelination deficit defines a novel interventional target for multiple system atrophy. Acta Neuropathol. 132, 59-75. doi: 10.1007/s00401-016-1572-y

Fabregat, A., Sidiropoulos, K., Viteri, G., Marin-Garcia, P., Ping, P., Stein, L., et al. (2018). Reactome diagram viewer: data structures and strategies to boost performance. Bioinformatics 34, 1208-1214. doi: 10.1093/bioinformatics/btx752

Franz, M., Lopes, C. T., Huck, G., Dong, Y., Sumer, O., and Bader, G. D. (2016). Cytoscape.js: a graph theory library for visualisation and analysis. Bioinformatics 32, 309-311. doi: 10.1093/bioinformatics/btv557

Goldberg, J., Daniel, M., van Heuvel, Y., Victor, M., Beyer, C., Clarner, T., et al. (2013). Short-term cuprizone feeding induces selective amino acid deprivation with concomitant activation of an integrated stress response in oligodendrocytes. Cell. Mol. Neurobiol. 33, 1087-1098. doi: 10.1007/s10571013-9975-y

Gururajan, A., Taylor, D. A., and Malone, D. T. (2011). Effect of cannabidiol in a MK-801-rodent model of aspects of schizophrenia. Behav. Brain Res. 222, 299-308. doi: 10.1016/j.bbr.2011.03.053

Hattori, T., Shimizu, S., Koyama, Y., Emoto, H., Matsumoto, Y., Kumamoto, N., et al. (2014). DISC1 (disrupted-in-schizophrenia-1) regulates differentiation of oligodendrocytes. PLoS One 9:e88506. doi: 10.1371/journal.pone.0088506

Hendouei, N., Farnia, S., Mohseni, F., Salehi, A., Bagheri, M., Shadfar, F., et al. (2018). Alterations in oxidative stress markers and its correlation with clinical findings in schizophrenic patients consuming perphenazine, clozapine and risperidone. Biomed. Pharmacother. 103, 965-972. doi: 10.1016/j.biopha.2018. 04.109

Huang, D. W., Sherman, B. T., and Lempicki, R. A. (2009a). Bioinformatics enrichment tools: paths toward the comprehensive functional analysis of large gene lists. Nucleic Acids Res. 37, 1-13. doi: 10.1093/nar/gkn923

Huang, D. W., Sherman, B. T., and Lempicki, R. A. (2009b). Systematic and integrative analysis of large gene lists using DAVID bioinformatics resources. Nat. Protoc. 4, 44-57. doi: 10.1038/nprot.2008.211

Hubler, Z., Allimuthu, D., Bederman, I., Elitt, M. S., Madhavan, M., Allan, K. C., et al. (2018). Accumulation of 8,9-unsaturated sterols drives oligodendrocyte formation and remyelination. Nature 560, 372-376. doi: 10.1038/s41586-0180360-3

Iwata, K., Café-Mendes, C. C., Schmitt, A., Steiner, J., Manabe, T., Matsuzaki, H., et al. (2013). The human oligodendrocyte proteome. Proteomics 13, 3548-3553. doi: 10.1002/pmic.201300201

Jinsmaa, Y., Isonaka, R., Sharabi, Y., and Goldstein, D. S. (2020). 3,4Dihydroxyphenylacetaldehyde is more efficient than dopamine in oligomerizing and quinonizing $\alpha$-synuclein. J. Pharmacol. Exp. Ther. 372, 157-165. doi: 10.1124/jpet.119.262246

Jørgensen, K. N., Nerland, S., Norbom, L. B., Doan, N. T., Nesvåg, R., MørchJohnsen, L., et al. (2016). Increased MRI-based cortical grey/white-matter contrast in sensory and motor regions in schizophrenia and bipolar disorder. Psychol. Med. 46, 1971-1985. doi: 10.1017/S0033291716000593

Kim, W., Hahn, K. R., Jung, H. Y., Kwon, H. J., Nam, S. M., Kim, J. W., et al. (2019). Melatonin ameliorates cuprizone-induced reduction of hippocampal neurogenesis, brain-derived neurotrophic factor and phosphorylation of cyclic AMP response element-binding protein in the mouse dentate gyrus. Brain Behav. 9:e01388. doi: 10.1002/brb3.1388

Leucht, S., Corves, C., Arbter, D., Engel, R. R., Li, C., and Davis, J. M. (2009). Second-generation versus first-generation antipsychotic drugs for schizophrenia: a meta-analysis. Lancet 373, 31-41. doi: 10.1016/S01406736(08)61764-X
Leweke, F. M., Piomelli, D., Pahlisch, F., Muhl, D., Gerth, C. W., Hoyer, C., et al. (2012). Cannabidiol enhances anandamide signaling and alleviates psychotic symptoms of schizophrenia. Transl. Psychiatry 2:e94. doi: 10.1038/tp.2012.15

Liebermeister, W., Noor, E., Flamholz, A., Davidi, D., Bernhardt, J., and Milo, R. (2014). Visual account of protein investment in cellular functions. Proc. Natl. Acad. Sci. U. S. A. 111, 8488-8493. doi: 10.1073/pnas.1314810111

Lotia, S., Montojo, J., Dong, Y., Bader, G. D., and Pico, A. R. (2013). Cytoscape app store. Bioinformatics 29, 1350-1351. doi: 10.1093/bioinformatics/ btt138

McGuire, P., Robson, P., Cubala, W. J., Vasile, D., Morrison, P. D., Barron, R., et al. (2018). Cannabidiol (CBD) as an adjunctive therapy in schizophrenia: a multicenter randomized controlled trial. Am. J. Psychiatry 175, 225-231. doi: 10.1176/appi.ajp.2017.17030325

McLaurin, J., Trudel, G. C., Shaw, I. T., Antel, J. P., and Cashman, N. R. (1995). A human glial hybrid cell line differentially expressing genes subserving oligodendrocyte and astrocyte phenotype. J. Neurobiol. 26, 283-293. doi: 10.1002/neu.480260212

Mecha, M., Torrão, A. S., Mestre, L., Carrillo-Salinas, F. J., Mechoulam, R., and Guaza, C. (2012). Cannabidiol protects oligodendrocyte progenitor cells from inflammation-induced apoptosis by attenuating endoplasmic reticulum stress. Cell Death Dis. 3:e331. doi: 10.1038/cddis.2012.71

Messori, L., Casini, A., Gabbiani, C., Sorace, L., Muniz-Miranda, M., and Zatta, P. (2007). Unravelling the chemical nature of copper cuprizone. Dalton Trans. 21, 2112-2114. doi: 10.1039/b701896g

Metsalu, T., and Vilo, J. (2015). ClustVis: a web tool for visualizing clustering of multivariate data using principal component analysis and heatmap. Nucleic Acids Res. 43, W566-W570. doi: 10.1093/nar/gkv468

O’Neill, L. A., and Kaltschmidt, C. (1997). NF-к B: a crucial transcription factor for glial and neuronal cell function. Trends Neurosci. 20, 252-258. doi: 10.1016/s0166-2236(96)01035-1

Orban, P., Desseilles, M., Mendrek, A., Bourque, J., Bellec, P., and Stip, E. (2017). Altered brain connectivity in patients with schizophrenia is consistent across cognitive contexts. J. Psychiatry Neurosci. 42, 17-26. doi: 10.1503/jpn.150247

Owen, M. J., Sawa, A., and Mortensen, P. B. (2016). Schizophrenia. Lancet 388, 86-97. doi: 10.1016/S0140-6736(15)01121-6

Petronilli, V., and Zoratti, M. (1990). A characterization of cuprizone-induced giant mouse liver mitochondria. J. Bioenerg. Biomembr. 22, 663-677. doi: 10.1007/BF00809070

Praet, J., Guglielmetti, C., Berneman, Z., Van der Linden, A., and Ponsaerts, P. (2014). Cellular and molecular neuropathology of the cuprizone mouse model: clinical relevance for multiple sclerosis. Neurosci. Biobehav. Rev. 47, 485-505. doi: 10.1016/j.neubiorev.2014.10.004

Rahimi, A., Faizi, M., Talebi, F., Noorbakhsh, F., Kahrizi, F., and Naderi, N. (2015). Interaction between the protective effects of cannabidiol and palmitoylethanolamide in experimental model of multiple sclerosis in C57BL/6 mice. Neuroscience 290, 279-287. doi: 10.1016/j.neuroscience.2015. 01.030

Sachs, H. H., Bercury, K. K., Popescu, D. C., Narayanan, S. P., and Macklin, W. B. (2014). A new model of cuprizone-mediated demyelination/remyelination. ASN Neuro 6:1759091414551955. doi: 10.1177/1759091414551955

Saito, Y., Kubicki, M., Koerte, I., Otsuka, T., Rathi, Y., Pasternak, O., et al. (2018). Impaired white matter connectivity between regions containing mirror neurons and relationship to negative symptoms and social cognition, in patients with first-episode schizophrenia. Brain Imaging Behav. 12, 229-237. doi: 10.1007/s11682-017-9685-Z

Sánchez-Wandelmer, J., Dávalos, A., de la Peña, G., Cano, S., Giera, M., Canfrán-Duque, A., et al. (2010). Haloperidol disrupts lipid rafts and impairs insulin signaling in SH-SY5Y cells. Neuroscience 167, 143-153. doi: 10.1016/j. neuroscience.2010.01.051

Santos, A. K., Vieira, M. S., Vasconcellos, R., Goulart, V. A. M., Kihara, A. H., and Resende, R. R. (2019). Decoding cell signalling and regulation of oligodendrocyte differentiation. Semin. Cell Dev. Biol. 95, 54-73. doi: 10.1016/j. semcdb.2018.05.020

Seabra, G., de Almeida, V., and Martins-de-Souza, D. (2019). Maturation of a human oligodendrocyte cell line. Methods Mol. Biol. 1916, 113-121. doi: 10.1007/978-1-4939-8994-2_11

Seabra, G., de Almeida, V., Reis-de-Oliveira, G., Crunfli, F., Antunes, A. S. L. M., and Martins-de-Souza, D. (2020). Ubiquitin-proteasome system, lipid 
metabolism and DNA damage repair are triggered by antipsychotic medication in human oligodendrocytes: implications in schizophrenia. Sci. Rep. 10:12655. doi: 10.1038/s41598-020-69543-5

Sun, S., Hu, F., Wu, J., and Zhang, S. (2017). Cannabidiol attenuates OGD/Rinduced damage by enhancing mitochondrial bioenergetics and modulating glucose metabolism via pentose-phosphate pathway in hippocampal neurons. Redox Biol. 11, 577-585. doi: 10.1016/j.redox.2016.12.029

Szilagyi, G. T., Nawrocki, A. M., Eros, K., Schmidt, J., Fekete, K., Elkjaer, M. L., et al. (2020). Proteomic changes during experimental de- and remyelination in the corpus callosum. PLoS One 15:e230249. doi: 10.1371/journal.pone. 0230249

Szklarczyk, D., Morris, J. H., Cook, H., Kuhn, M., Wyder, S., Simonovic, M., et al. (2017). The STRING database in 2017: quality-controlled proteinprotein association networks, made broadly accessible. Nucleic Acids Res. 45, D362-D368. doi: 10.1093/nar/gkw937

Taraboletti, A., Walker, T., Avila, R., Huang, H., Caporoso, J., Manandhar, E., et al. (2017). Cuprizone intoxication induces cell intrinsic alterations in oligodendrocyte metabolism independent of copper chelation. Biochemistry 56, 1518-1528. doi: 10.1021/acs.biochem.6b01072

Templeton, N., Kivell, B., McCaughey-Chapman, A., Connor, B., and La Flamme, A. C. (2019). Clozapine administration enhanced functional recovery after cuprizone demyelination. PLoS One 14:e0216113. doi: 10.1371/journal. pone. 0216113

Thompson, K. K., Nissen, J. C., Pretory, A., and Tsirka, S. E. (2018). Tuftsin combines with remyelinating therapy and improves outcomes in models of CNS demyelinating disease. Front. Immunol. 9:2784. doi: 10.3389/fimmu.2018. 02784

van Os, J., and Kapur, S. (2009). Schizophrenia. Lancet 374, 635-645. doi: 10.1016/S0140-6736(09)60995-8

Venturini, G. (1973). Enzymic activities and sodium, potassium and copper concentrations in mouse brain and liver after cuprizone treatment in vivo. J. Neurochem. 21, 1147-1151. doi: 10.1111/j.1471-4159.1973.tb07569.x

Vikhreva, O. V., Rakhmanova, V. I., Orlovskaya, D. D., and Uranova, N. A. (2016). Ultrastructural alterations of oligodendrocytes in prefrontal white matter in schizophrenia: a post-mortem morphometric study. Schizophr. Res. 177, 28-36. doi: 10.1016/j.schres.2016.04.023

Werner, S. R., Saha, J. K., Broderick, C. L., Zhen, E. Y., Higgs, R. E., Duffin, K. L., et al. (2010). Proteomic analysis of demyelinated and remyelinating brain tissue following dietary cuprizone administration. J. Mol. Neurosci. 42, 210-225. doi: 10.1007/s12031-010-9354-9

Xu, H., Yang, H.-J., and Li, X.-M. (2014). Differential effects of antipsychotics on the development of rat oligodendrocyte precursor cells exposed to cuprizone. Eur. Arch. Psychiatry Clin. Neurosci. 264, 121-129. doi: 10.1007/s00406-0130414-3

Xu, Z., Adilijiang, A., Wang, W., You, P., Lin, D., Li, X., et al. (2019). Arecoline attenuates memory impairment and demyelination in a cuprizone-induced mouse model of schizophrenia. NeuroReport 30, 134-138. doi: 10.1097/WNR. 0000000000001172

Yoo, D. Y., Cho, S. B., Jung, H. Y., Kim, W., Lee, K. Y., Kim, J. W., et al. (2019). Protein disulfide-isomerase A3 significantly reduces ischemia-induced damage by reducing oxidative and endoplasmic reticulum stress. Neurochem. Int. 122, 19-30. doi: 10.1016/j.neuint.2018.11.002

Zahednasab, H., Firouzi, M., Kaboudanian-Ardestani, S., Mojallal-Tabatabaei, Z., Karampour, S., and Keyvani, H. (2019). The protective effect of rifampicin on behavioral deficits, biochemical and neuropathological changes in a cuprizone model of demyelination. Cytokine 113, 417-426. doi: 10.1016/j.cyto.2018. 10.016

Zajicek, J. P., and Apostu, V. I. (2011). Role of cannabinoids in multiple sclerosis. CNS Drugs 25, 187-201. doi: 10.2165/11539000-000000000-00000

Zhang, X., Lan, Y., Xu, J., Quan, F., Zhao, E., Deng, C., et al. (2019). CellMarker: a manually curated resource of cell markers in human and mouse. Nucleic Acids Res. 47, D721-D728. doi: 10.1093/nar/gky900

Zhou, Y., Zhou, B., Pache, L., Chang, M., Khodabakhshi, A. H., Tanaseichuk, O., et al. (2019). Metascape provides a biologist-oriented resource for the analysis of systems-level datasets. Nat. Commun. 10:1523. doi: 10.1038/s41467-01909234-6

Zuardi, A. W., Crippa, J. A. S., Hallak, J. E. C., Bhattacharyya, S., Atakan, Z., Martin-Santos, R., et al. (2012). A critical review of the antipsychotic effects of cannabidiol: 30 years of a translational investigation. Curr. Pharm. Des. 18, 5131-5140. doi: 10.2174/138161212802884681

Conflict of Interest: JC is a member of the International Advisory Board of the Australian Centre for Cannabinoid Clinical and Research Excellence (ACRE)-National Health and Medical Research Council (NHMRC). JC and $\mathrm{JH}$ have received travel support to attend scientific meetings and personal consultation fees from BSPG-Pharm. JC, JH, and AZ are co-inventors of the patent "Fluorinated CBD compounds, compositions and uses thereof. Pub. No. WO/2014/108899. International Application No.: PCT/IL2014/050023," Def. US number Reg. 62193296; July 29, 2015; INPI on August 19, 2015 (BR1120150164927; Mechoulam R, Zuardi AW, Kapczinski F, Hallak JEC, Guimarães FS, Crippa JAS, Breuer A). Universidade de São Paulo (USP) has licensed this patent to Phytecs Pharm (USP Resolution No. 15.1.130002.1.1) and has an agreement with Prati-Donaduzzi to "develop a pharmaceutical product containing synthetic CBD and prove its safety and therapeutic efficacy in the treatment of epilepsy, schizophrenia, Parkinson's disease, and anxiety disorders." $\mathrm{JC}, \mathrm{JH}$, and $\mathrm{AZ}$ are co-inventors of the patent "Cannabinoid-containing oral pharmaceutical composition, method for preparing and using same," INPI on September 16, 2016 (BR 112018005423-2).

The remaining authors declare that the research was conducted in the absence of any commercial or financial relationships that could be construed as a potential conflict of interest.

Copyright $\odot 2021$ Falvella, Smith, Silva-Costa, Valença, Crunfli, Zuardi, Hallak, Crippa, Almeida and Martins-de-Souza. This is an open-access article distributed under the terms of the Creative Commons Attribution License (CC BY). The use, distribution or reproduction in other forums is permitted, provided the original author(s) and the copyright owner(s) are credited and that the original publication in this journal is cited, in accordance with accepted academic practice. No use, distribution or reproduction is permitted which does not comply with these terms. 